\title{
Significant new planetary nebula discoveries as powerful probes of the $\mathrm{LMC}$
}

\author{
Warren A. Reid ${ }^{1}$ and Quentin A. Parker ${ }^{1,2}$ \\ ${ }^{1}$ Dept of Physics, Macquarie University North Ryde, Sydney, NSW 2190, Australia \\ email: warren@ics.mq.edu.au \\ ${ }^{2}$ Anglo-Australian Observatory, PO Box 296 Epping, NSW 1710, Australia \\ email: qap@ics.mq.edu.au
}

\begin{abstract}
Our discovery and analysis of 452 new planetary nebulae (PNe) in the Large Magellanic Cloud (LMC) has tripled the number of known LMC PNe, providing a powerful new resource for probing the kinematics of the LMC as well as contributing fresh insight into the PN luminosity function (PNLF) which we now extend to over 10 magnitudes in [O III] and $\mathrm{H} \alpha$. These discoveries have resulted from a new, deep $(R \equiv 22)$, high resolution $\mathrm{H} \alpha$ map of the central $25 \mathrm{deg}^{2}$ of the LMC, achieved by a process of multi-exposure median co-addition of a dozen 2-hour exposures. The resulting map is at least 1 magnitude deeper than the best wide-field narrow-band LMC images currently available and has proven a major resource for the discovery of emission objects of all kinds. As a result, the near complete sample of the PN population in the central $25 \mathrm{deg}^{2}$ of the LMC has permitted truly meaningful quantitative determinations of the PNLF, distribution, abundances and kinematics. We briefly describe the importance of these PN discoveries, the additional spectroscopic confirmation of $>2,000$ compact emission sources, flux calibration, the newly derived electron temperatures and electron densities.
\end{abstract}

Keywords. surveys, planetary nebulae: general, galaxies: individual (LMC), Magellanic Clouds

\section{Introduction}

Planetary nebulae $(\mathrm{PNe})$ are a short-lived phase in the late evolution of low mass stars and are important astrophysical tools. They provide key data on the physics of stellar evolution, mass loss (Iben 1995), nucleosynthesis processes, abundance gradients and ISM chemical enrichment. They are powerful tracers of star-forming history (e.g., Maciel \& Costa 2003). Accurate velocities and nebular parameters, such as excitation class and electron temperature, can be derived from their strong emission lines which are detectable at large distances. Most physical PNe properties, including ionized and total nebular masses and the brightness and evolutionary states of their central stars, depend on accurate distances (Ciardullo \& Jacoby 1999). This is difficult in our own Galaxy due to inherent problems with variable extinction and lack of central star homogeneity (Terzian 1997). The well determined 50.6 kpc LMC distance (e.g., Keller \& Wood 2006), modest 35 degree inclination angle and disk thickness (only $\sim 500 \mathrm{pc}$, van der Marel \& Cioni 2001), mean that all LMC PNe are effectively co-located. Since dimming of their light by intervening gas and dust is low and uniform (e.g., Kaler \& Jacoby 1990), we can better estimate absolute nebula luminosity and size.

\section{An unprecedented catalogue of LMC PNe}

Reid \& Parker (2006b) (hereafter RPb) have constructed the most complete, least biased census of a PNe population ever compiled for a single galaxy based on discoveries from their deep $A A O / U K S T \mathrm{H} \alpha$ multi-exposure stack of the LMC's central $25 \mathrm{deg}^{2}$ 
(Reid \& Parker 2006a). This stack comprises a series of 12 repeated narrow-band 'A'grade $\mathrm{H} \alpha$ and 6 matching broad-band 'SR' (Short Red) exposures of the central LMC field, taken over a three year period. From these exposures, deep, homogeneous, narrowband $\mathrm{H} \alpha$ and matching broad-band 'SR' maps of the entire central $25 \mathrm{deg}^{2}$ square of the LMC were constructed. Using these maps, over 2,000 emission objects in the central area of the LMC were identified. A major spectral confirmation program was undertaken in November and December 2004 mainly comprising 5 nights using $2 \mathrm{dF}$ on the Anglo-Australian Telescope but supported with 7 nights using the $1.9 \mathrm{~m}$ at the South African Astronomical Observatory, 3 nights using the FLAMES multi-object spectrograph on the ESO Very Large Telescope, 7 nights using the $2.3 \mathrm{~m}$ Australian National University telescope at the Siding Spring Observatory and 3 half nights using $6 \mathrm{dF}$ on the UKST.

Individual exposure times for the $182 \mathrm{dF}$ fields observed were $1200 \mathrm{~s}$ using the $300 \mathrm{~B}$ grating with a central wavelength of $5852 \AA$ and wavelength range $3600-8000 \AA$ at a dispersion of $4.30 \AA$ /pixel. These low-resolution observations (9.0 $\AA$ FWHM) were used as the primary means of object classification. All fields were re-observed using the 1200R high resolution grating with a central wavelength of $6793 \AA$. These observations covered a range $6200-7300 \AA$ with a dispersion of $1.10 \AA /$ pixel and resolution of $2.2 \AA$ FWHM which cleanly separated the [S II] 6716 and 6731 lines used for electron density determination. The high resolution spectra were also used for determination of accurate velocities (see $\mathrm{RPb}$ ). In all we had 7,521 high and low resolution object spectra for LMC targets.

A combination of spectroscopy and image analysis confirmed 452 new PN candidates along with the 161 previously known PNe in the survey area. A large fraction of new LMC PNe are $\geqslant 3 \times$ fainter than those previously known, effectively tripling numbers accrued from all surveys over the last 80 years. These additional objects have already led to significant advances in our understanding of the kinematical sub-structure of the central LMC including rotation, inclination and transverse velocity as well as the distribution of the old stellar population $(\mathrm{RPb})$. They will assist us in refining the PNLF and physical characteristics such as temperatures, densities, nebulae masses and abundances (Reid 2008). It is our intention to publish a detailed analysis within the next 12 months.

\section{Objects discovered}

Table 1 provides a summary of our object classification following spectral analysis. It includes 2 previously known PNe now re-classified as $\mathrm{H}$ II regions, a further 4 demoted to possible $\mathrm{PNe}$ and 1 to likely $(\mathrm{RPb})$. With the exception of previously known PNe, the numbers for other previously known objects represent those included in the catalogue and observed. They do not necessarily represent the full number for that object type that may exist within the central $25 \mathrm{deg}^{2}$ of the LMC. Figure 1 shows an example of 6 new LMC PNe we have classified as 'true', due to their strong [O III] $/ \mathrm{H} \beta$ ratios, lack of relatively strong continuum and clean separation from neighbouring stars. Careful reexamination of object images and spectra with the addition of IRAC false colour images from SAGE (Spitzer) and new high resolution radio mosaic images from Parks/ATCA have assisted us in the re-classification of several possible and likely PNe. We have reclassified RP 1495, RP 1716, RP 872, RP 1113, RP 641, RP 105, RP 1933 as compact H II regions. The high resolution Spitzer images have also revealed 2 objects in the position of RP 1534. We classify the larger one to the NW as a bright H II region with a central star. The PN is clearly separated ( $\sim 2$ arcsec) to the SE. In the same way, SMP 48 also clearly 
Table 1. Emission object classification results from spectral observations covering the central $25 \mathrm{deg}^{2}$, area of the main LMC bar.

\begin{tabular}{lcc}
\hline Object & $\begin{array}{c}\text { Previously } \\
\text { Known }\end{array}$ & $\begin{array}{c}\text { Newly } \\
\text { Confirmed }\end{array}$ \\
\hline PNe "True" & $162(-2)$ & 285 \\
PNe "Likely" & 1 & 53 \\
PNe "Possible" & 4 & 114 \\
Emission-line stars & 55 & 622 \\
Late-type stars & 10 & 247 \\
H II regions & 85 & 70 \\
S/N too low for ID & & 32 \\
Variable stars & 61 & 28 \\
Emission objects of unknown nature & 12 & 25 \\
SNR & 9 & 18 \\
Wolf-Rayet stars & 14 & 8 \\
\hline
\end{tabular}
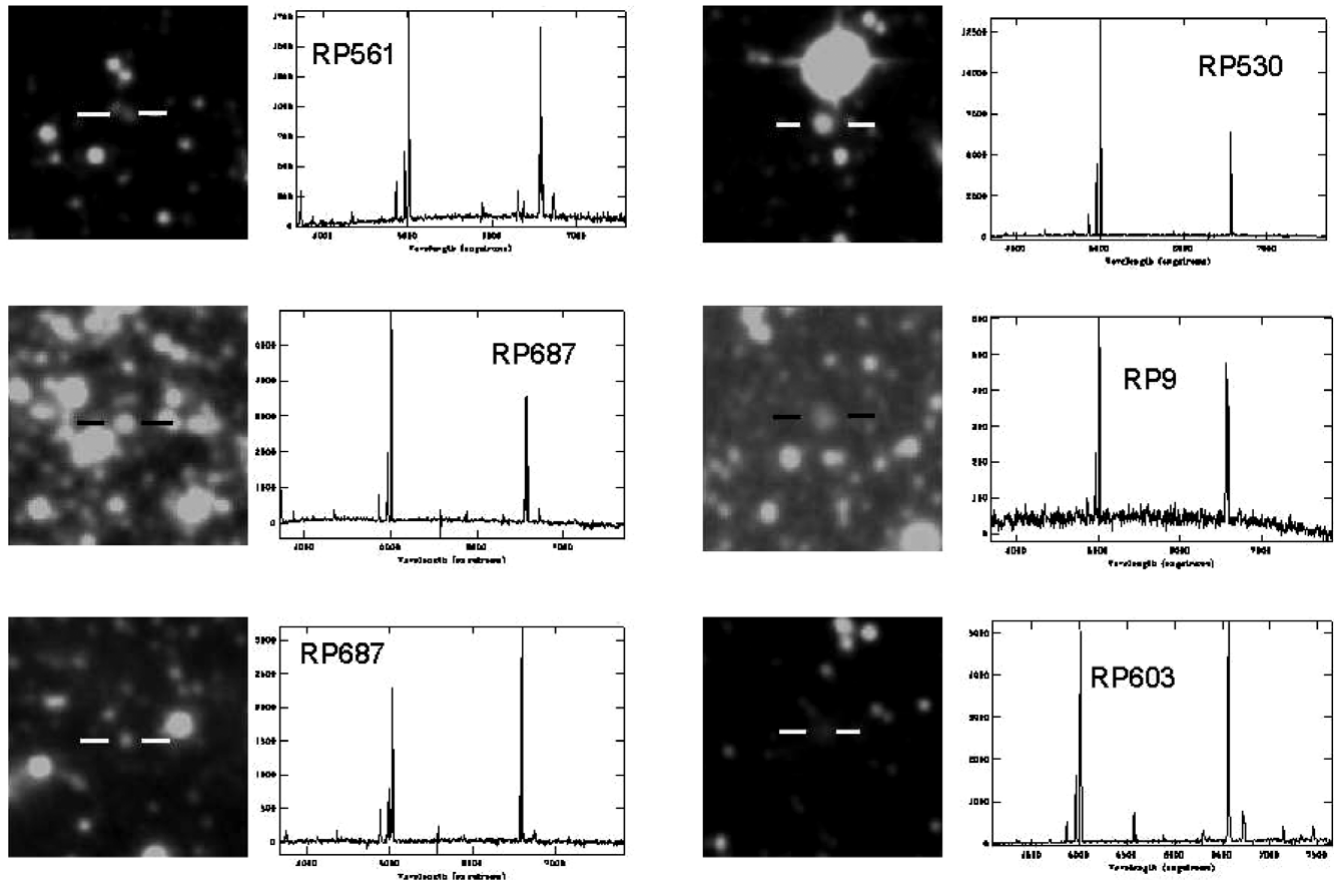

Figure 1. Six examples of PNe discovered in the RP survey of the LMC showing strong $[\mathrm{O}$ III $] / \mathrm{H} \beta$ ratios.

separates into 2 equal size objects. The object to the east is an $\mathrm{H}$ II region while the PN is to the west. This demonstrates the power of multiwavelength data in the identification process (e.g., Parker et al. 2006).

\section{Flux calibration}

Flux calibration of the new LMC PN spectra was a prerequisite for determining temperatures, densities, nebula masses and chemical abundances. It also facilitated conversion 
to magnitudes for luminosity studies. To achieve this, a flux calibration technique had to be found. This has previously proved quite difficult to do with fibre-based spectra.

As each of the $182 \mathrm{dF}$ field-plate exposures has its own relative line strengths and creates its own individual trend across the spectrum, experiments were undertaken in order to find the best method of flux calibration using the observed line intensities. The best results were obtained by individually calibrating each spectral line on each field plate to raw MCPN fluxes gained from HST exposures (Shaw, private communication). The known PNe included on each field plate were used as flux calibrators for each individual field. The MCPN fluxes for known PNe observed on each field plate exposure were graphed against the individual $2 \mathrm{dF}$ line intensities. In each case, a line of best fit was derived and the underlying linear equation extracted. This equation became the calibrator for each emission line on each individual $2 \mathrm{dF}$ field plate exposure. The equation was applied to each spectrum with a detectable line intensity in that field.

Fluxes for LMC PNe from other catalogues (Jacoby et al. 1990; Leisy et al. private communication; Meatheringham et al. 1988) were also included in order to build up the number of calibrators per field. Where only a de-reddened flux value was published, a relative reddening was applied in order to make a better correlation. These fluxes were used for double checking since each independent survey revealed many fluxes which agreed and equally many which varied considerably. A spread in published line fluxes for the same PN is common but helps us to establish the uncertainties. Since the MCPN catalogue contained the largest number of PNe and the raw fluxes were 'as measured' (not de-reddened) these were given preference where irregularities became obvious. The MCPN set also includes some ground-based fluxes from ELCAT available at http://stsdas.stsci.edu/elcat/ where the spectra are uncorrected for extinction. Following flux calibration, a number of nebula diagnostic values such as temperatures, densities and ionised masses were derived. Sections 5 and 6 give a brief introduction to some of our results.

\section{Determination of nebula electron temperature}

The measurement of electron temperatures $\left(T_{\mathrm{e}}\right)$ from ions in PNe is important not only as a diagnostic for understanding the photoionisation of the nebulae but is required for subsequent density and abundance determinations. In PNe, the most powerful mechanism for the loss of energy by free electrons is the excitation of forbidden lines. A $T_{\mathrm{e}}$ based on these lines is an independent parameter describing conditions in the nebula. $T_{\mathrm{e}}$ is not a strong function of the distance to the central ionising star (Stasińska 2002). It is, however, higher for high stellar temperatures $\left(T_{\star}\right)$ and low metallicity values, $Z$. The [O III $] 4363,5007 \AA$ ratio is sensitive to metallicity and effective $T_{\star}$. At higher metallicity, the nebulae are cooled efficiently through the [O III] 5007 line, producing cooler nebulae (Osterbrock \& Ferland 2006). For $\mathrm{O}^{++}$, the transition from level ${ }^{1} S_{0}$ to the ${ }^{1} D_{2}$ level emits the forbidden line [O III] 4363. The transition from ${ }^{1} D_{2}$ downward to the levels ${ }^{3} P_{1,2}$ creates the so called $\mathrm{N}_{1}$ and $\mathrm{N}_{2}$ 'nebulium' ([O III $] 4959 \AA$ and $5007 \AA$ ) lines. An estimate of electron temperature can then be made by comparing the number of ions in the ${ }^{1} S_{0}$ state with the number in the ${ }^{1} D_{2}$ (i.e. the [O III] 4363 increase in strength over [O III] $\left(\mathrm{N}_{1}+\mathrm{N}_{2}\right)$ (Osterbrock 1989). However, in many PNe, this is not straightforward to measure as the $4363 \AA$ line can range between a hundredth and a thousandth the strength of the $5007 \AA$ line. The method applied was the temperature equation provided by Osterbrock \& Ferland (2006), p. 109.

Although the [N II] $5755 \AA$ line can also be very faint, it was clearly measurable for 159 PNe. The $[\mathrm{N} \mathrm{II}](6548 \AA+6583 \AA) / 5755 \AA$ calculation provided us with an alternative temperature estimate (preferable to default values) for density determinations in PNe 


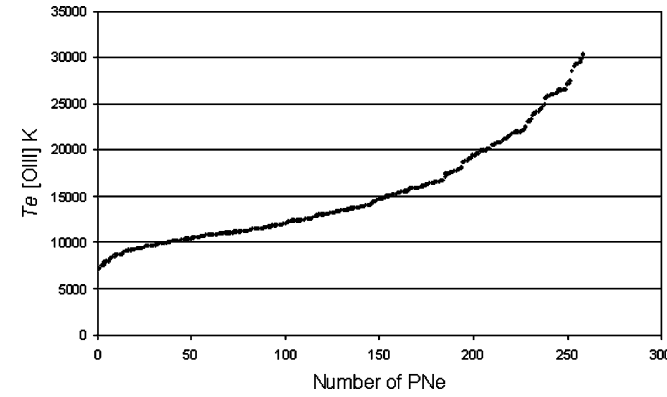

Figure 2. The derived electron temperatures for all $256 \mathrm{LMC} \mathrm{PNe}$ with measurable lines of [O III] $4363 \AA, 4959 \AA$ and $5007 \AA$. Temperatures range from $7,728 \mathrm{~K}$ to 30,107 $\mathrm{K}$ with $80 \%$ of $\mathrm{PNe}$ under $20,000 \mathrm{~K}$.

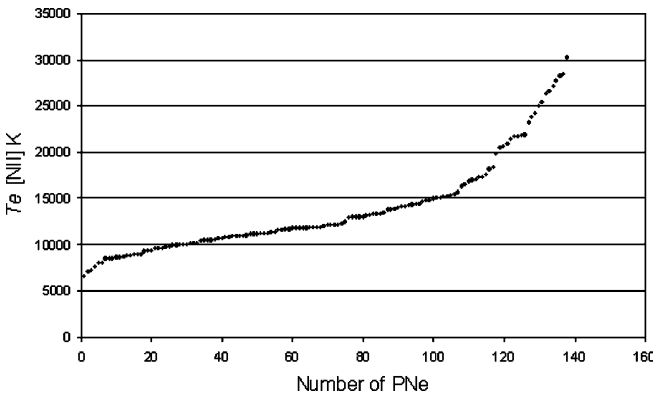

Figure 3. The derived electron temperatures for all $137 \mathrm{LMC}$ PNe with measurable lines of [N II] $5755 \AA$, $6548 \AA$ and $6583 \AA$. Temperatures range from $6,646 \mathrm{~K}$ to 30,099 $\mathrm{K}$ with $85 \%$ of $\mathrm{PNe}$ under $20,000 \mathrm{~K}$.

where [O III] 4363 was unavailable to provide an [O III] temperature. The $T_{\mathrm{e}}$ using [N II] was primarily calculated for use in the low zone abundance determinations following in the form of Osterbrock \& Ferland (2006), p. 193.

Figures 2 and 3 show the resulting $T_{\mathrm{e}}$ for [O III] and [ $\left.\mathrm{N} \mathrm{II}\right]$ respectively. High $T_{\mathrm{e}}$ between 20,000 and $30,000 \mathrm{~K}$ are due to both the high $T_{\star}$ of the PN and high electron densities in PNe, compared to H II regions as well as low $Z$ in the LMC. Some of the large dispersion is also due to physical differences in the nebulae and may also be related to nebula evolution (e.g., Zhang et al. 2004). $108 \mathrm{PNe}$ or $43 \%$ of the [O III] $T_{\mathrm{e}}$ sample are above 15,000 $\mathrm{K}$ (generally the highest $T_{\mathrm{e}}$ for $\mathrm{H}$ II regions (Osterbrock \& Ferland 2006)). This compares to only $37 \mathrm{PNe}$ or $28 \%$ yielding high [N II] $T_{\mathrm{e}}$. The lower percentage of high $T_{\mathrm{e}}$ in $[\mathrm{N}$ II] may be due to this line arising in the outer, cooler zones of the nebulae.

\section{Determination of Nebula Electron Density}

Nebula electron density $\left(n_{\mathrm{e}}\right)$ is one of the key parameters needed to confidently derive chemical abundances of nebulae and to calculate the total ionised mass used in certain distance/radius relation formulae. The $[\mathrm{S}$ II] doublet method, using the singly ionized sulphur lines at $\lambda 6731$ and $\lambda 6717$, as given by Osterbrock \& Ferland (2006), was employed to calculate $n_{\mathrm{e}}$. This doublet is emitted at the transition from level ${ }^{2} D_{3 / 2,5 / 2}$ to ${ }^{4} S_{3 / 2}$. The dependence of $n_{\mathrm{e}}$ and $T_{\mathrm{e}}$ on the ratio of $\lambda 6717 / \lambda 6731$ is given by:

$$
\frac{I \lambda 6717}{I \lambda 6731}=1.5 \frac{1+0.35 x}{1+0.96 x}, \quad \text { where } \quad x=10^{-2} \frac{n_{\mathrm{e}}}{T_{\mathrm{e}}^{1 / 2}} .
$$

The $[\mathrm{S} \mathrm{II}]$ lines were measured from the PN spectra obtained with the $2 \mathrm{dF} 1200 \mathrm{R}$ high resolution grating. The 1200R measurements were preferred for density estimates because the lines are always cleanly separated and no de-blending was required. Also, the [S II] lines are close in wavelength so the issue of flux calibration effects does not arise. The resulting histogram is shown in Figure 4. For comparison, the measured line intensities were also given as input to the IRAF STDAS temden task (Shaw \& Dufour 1995), which calculated the densities using the electron temperatures previously derived. The resulting densities were effectively equivalent to those derived using equation 6.1. Further analysis 


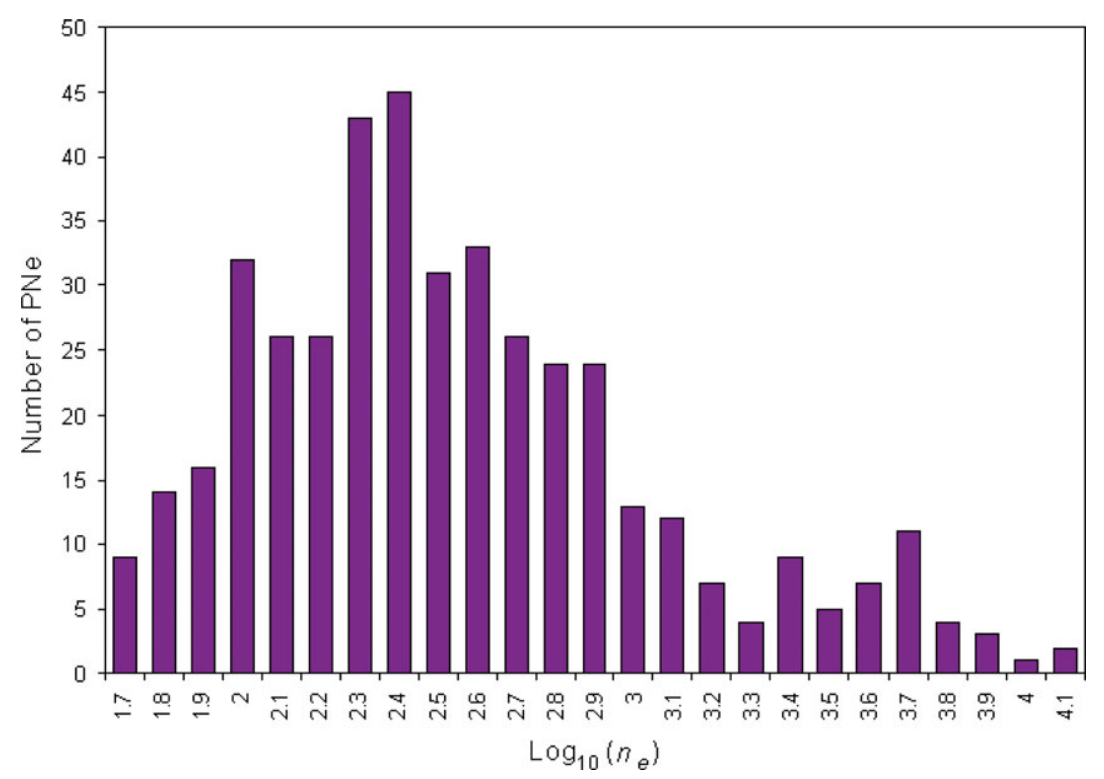

Figure 4. Histogram of electron densities for 487 LMC PNe. This is the largest and most comprehensive set of derived densities ever assembled for a PN sample. The range in the histogram is from 1.7 to $4.1 \mathrm{log}$ density with a peak at 2.4 embraced by a gradual rise and fall from 1.7 to $3.3 \mathrm{log}$ density. Of special interest is the second rise in the number of PNe between $\log 3.4$ and 4.1. These PNe are amongst the brightest in $\mathrm{H} \alpha$.

of these PNe, including nebula masses, excitation classes and a new luminosity function will be available shortly.

\section{References}

Ciardullo R. \& Jacoby, G. H. 1999, ApJ, 515, 191

Iben I . Jr. 1995, Phys.Reports, 250, 2

Jacoby G. H., Walker A. R., \& Ciardullo R. 1990, ApJ, 365, 471

Kaler J. B. \& Jacoby G. H. 1990, ApJ, 362, 491

Keller S. C. \& Wood, P. R. 2006, ApJ, 642, 834

Maciel W. J. \& Costa R. D. 2003, in S. Kwok, M. Dopita, \& R. Sutherland (eds.), Planetary Nebulae: Their Evolution and Role in the Universe, IAU Conf.Proc. 209 (ASP), p. 551

Meatheringham S. J., Dopita M. A., \& Morgan D. H. 1988, ApJ, 329, 166

Osterbrock, D. E. 1989, Astrophysics of Gaseous Nebulae and Active Galactic Nuclei (Mill Valley: University Science Books), p. 118

Osterbrock, D. E. \& Ferland, G. J. 2006, Astrophysics of Gaseous Nebulae and Active Galactic Nuclei (Sausalito, CA: University Science Books)

Parker Q. A., Acker A., Frew D. J., \& Reid, W. A. 2006, in M.J. Barlow \& R.H. Méndez (eds.), Planetary Nebulae in our Galaxy and Beyond, IAU Conf.Proc. 234 (Cambridge: CUP), p. 1

Reid, W. A. \& Parker Q. A. 2006a, MNRAS, 365, 401

Reid, W. A. \& Parker Q. A. 2006b, MNRAS, 373, 521

Reid W. A. 2008, PhD Thesis, Macquarie University, Sydney

Shaw, R. A. \& Dufour, R. J. 1995, PASP, 107, 896

Stasińska G. 2002, Rev. Mexicana AyA 12, 62

Terzian, Y. 1997, in H. J. Habing \& H. J. G. L. M. Lamers (eds.), Planetary Nebulae, IAU Conf.Proc. 180 (Dordrecht: Kluwer), p. 29

van der Marel, R. \& Cioni, M. 2001, AJ, 122, 1807

Zhang Y., Liu X.-W., Wesson R., Storey P. J., Liu Y., \& Danziger I. J. 2004, MNRAS, 351, 935 


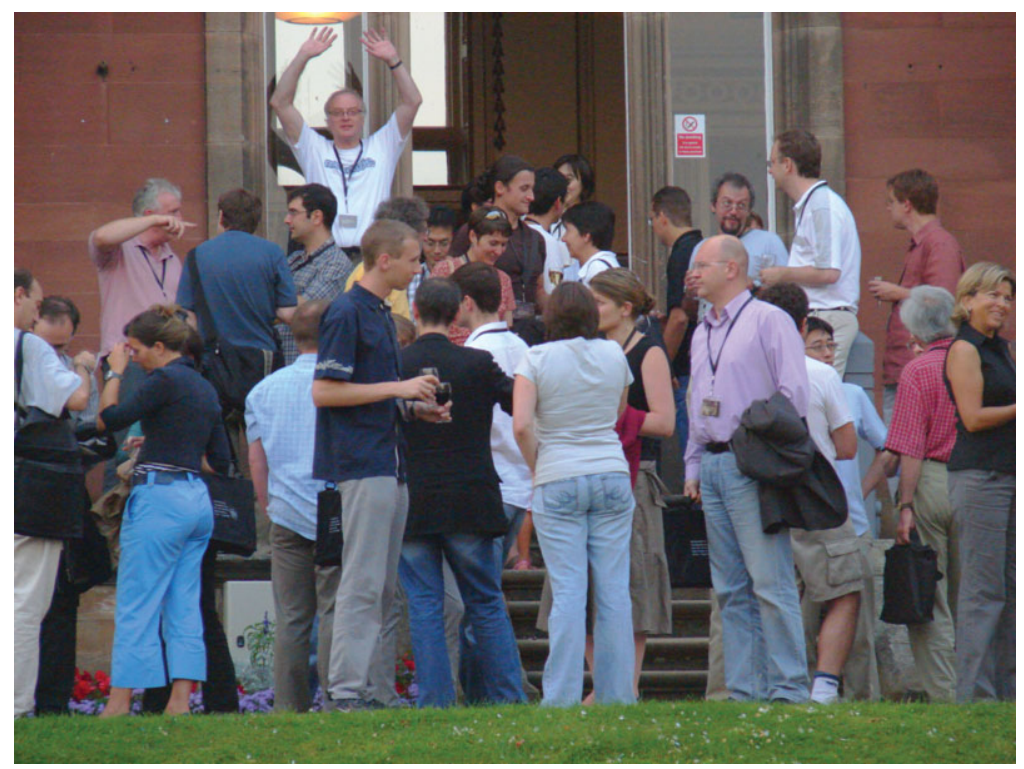

Nye Evans trying to initiate a Mexican wave, of which the others are blissfullly unaware.

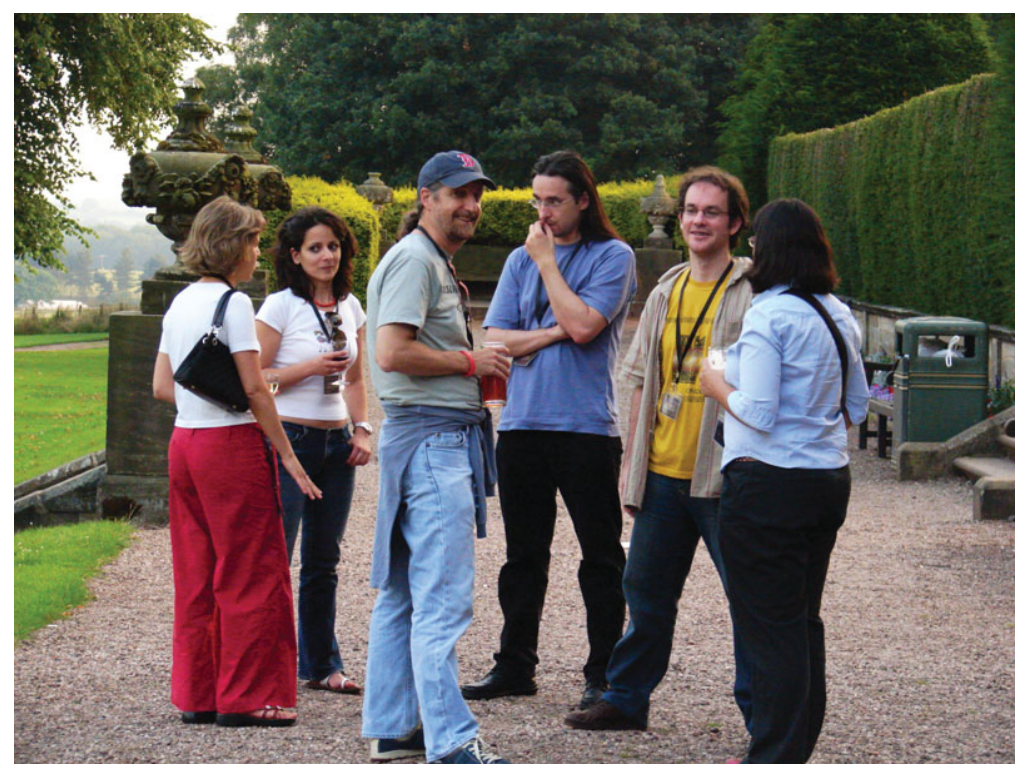

Enjoying a relaxed Summer's evening outside Keele Hall. 